\title{
Hybrid Trauma Management: Is It the Way We Are Heading?
}

\author{
Maya Paran MD and Boris Kessel MD \\ 'Surgical division, Hillel Yaffe Medical Center, affliated with Rappaport Medical School, Technion, Haifa, Israel
}

\begin{abstract}
Background: Hybrid trauma management, namely the combination of open and endovascular techniques and/or the application of endovascular methods in the operating/emergency room, is a quickly growing concept worldwide. However, its implications are not well established. We aimed to review the current data regarding hybrid trauma management in medical literature.

Methods: A review of the medical literature published between 2000 and 2020 using PubMed, Cochrane, Embase and Medline databases was performed in search of clinical studies regarding hybrid trauma treatments. Casereports were excluded from this review. The manuscripts were analyzed regarding the mechanism, location, and type of injury, endovascular and surgical techniques utilized, and the outcome.

Results: In total, 14 studies reporting hybrid trauma management in a total of 1,049 patients met the inclusion criteria and were analyzed. Blunt trauma was the leading trauma mechanism (87.1\%) and the most common procedure was transcatheter arterial embolization, performed in $29.7 \%$ of patients. The overall mortality was $15.2 \%$. Regarding case-control studies, $85.7 \%$ have shown hybrid trauma management to be associated with a shorter time from arrival to intervention, $42.9 \%$ reported lower rates of unfavorable outcome, and $28.6 \%$ reported reduced requirement for red blood cell transfusion as compared with conventional management.

Conclusions: Accumulating data suggests that hybrid management may be associated with a shorter time from arrival to intervention, lower rates of unfavorable outcomes and a reduced requirement for red blood cell transfusion as compared with conventional management of trauma patients.
\end{abstract}

Keywords: EVTM; Hybrid Trauma Management; Hybrid ER; Hybrid OR

\section{INTRODUCTION}

The concept of endovascular resuscitation for trauma management represents an attractive alternative treatment paradigm for trauma cases previously treated with open surgery $[1,2]$. Percutaneous trauma procedures may be used to achieve rapid hemorrhage control and urgent repair of damaged vessels [3]. Recent publications have demonstrated lower complication and mortality rates among patients treated by endovascular techniques [4-6].

Hybrid emergency and operating rooms have been reported in patient management in different medical fields, including management of cerebrovascular disease

\section{Corresponding author:}

Maya Paran MD, Hillel Yaffe Medical Center, Ha-Shalom St., PO Box 169, Hadera 3846201, Israel.

Email: paran.maya@gmail.com

(C) 2020 CC BY 4.0 - in cooperation with Depts. of Cardiothoracic/ Vascular Surgery, General Surgery and Anesthesia, Örebro University Hospital and Örebro University, Sweden
$[7,8]$, cardiac surgery $[9,10]$, and orthopedic surgery [11]. Utilizing hybrid rooms for the management of trauma patients is a new, rapidly progressing concept. Management of hemorrhage, in the setting of acute trauma, by endovascular and combined open-endo techniques has been reported frequently over the past few years [12]. This new approach, used for both hemodynamically stable and unstable patients, is named endovascular resuscitation and trauma management (EVTM) [12]. The foundation of the EVTM society in 2017 enables the sharing of information on advanced bleeding control methods and thus plays an important role in the evolution and growth of the field of hybrid trauma management. The management of trauma patients in different types of hybrid emergency and operating rooms has been described, including management of subclavian artery injury [13], innominate artery injury [14], aortic rupture [15], and tracheobronchial injury [16]. Due to the promising results of hybrid trauma management, installation of newly developed hybrid rooms for trauma management has been reported in recent years [17-19].

These hybrid rooms may eliminate the need to choose between interventional radiology techniques and surgical 


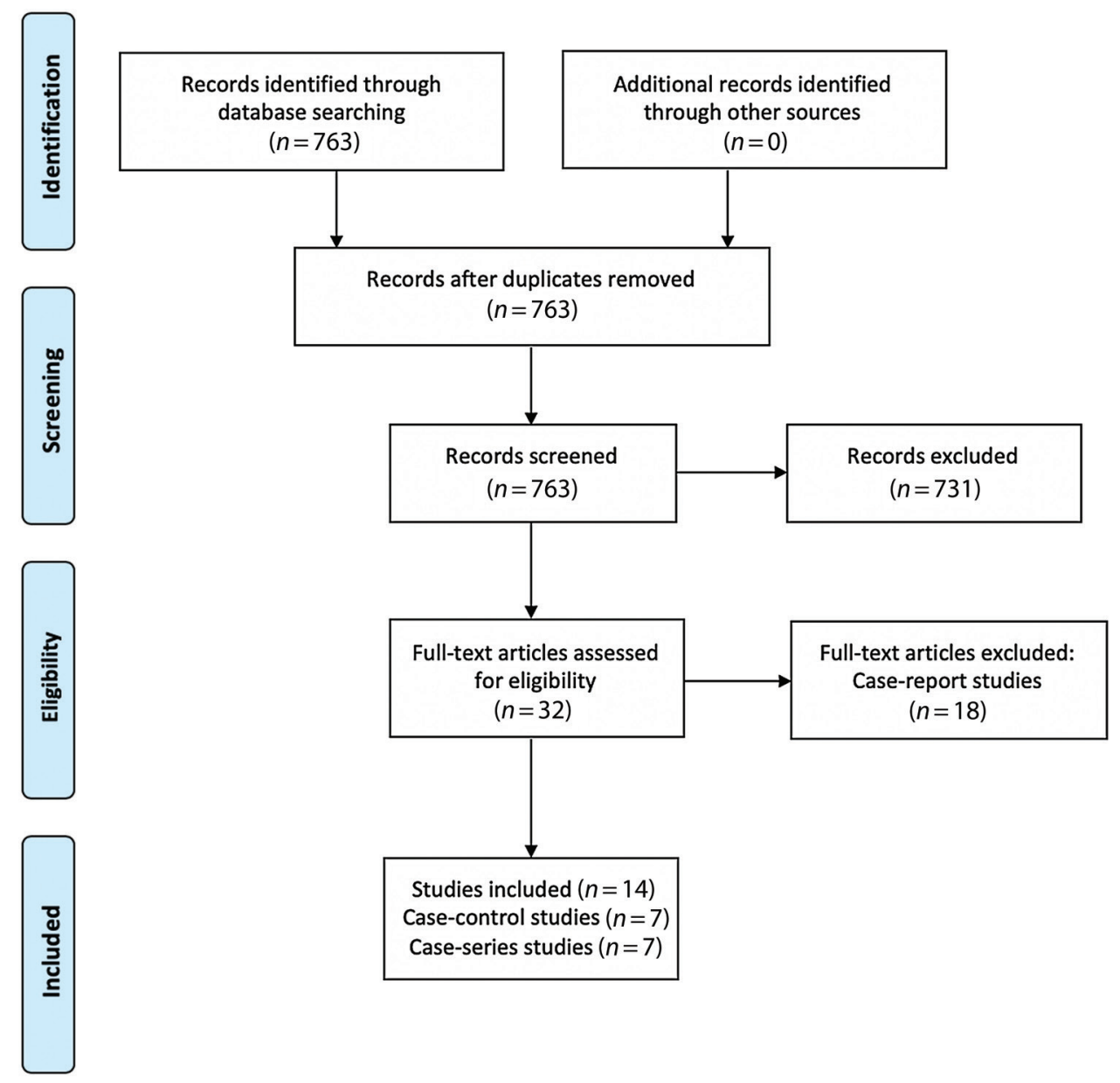

Figure 1 Flow chart of search results. $n=$ number of studies.

management and enable the management of trauma patients with combined definitive trauma interventions in a single suite. In addition, hybrid rooms may shorten the time interval from arrival to intervention in acute trauma patients by elimination of transfer time from the resuscitation room to an intervention suite [20]. Fehr et al. [20] assessed the potential benefit of hybrid trauma management and found that up to $7 \%$ of persistently hypotensive trauma patients may benefit from the utilization of a hybrid room for trauma management.

However, the hospital preparedness for hybrid trauma management requires sophisticated and expensive equipment, high-level professional skills, and commitment [21]. In addition, management of trauma patients in a hybrid room, combining open and endovascular techniques, is a concept that is still not well established and evidence supporting this type of management is relatively limited.

The aim of this review was to assess the evidence of advantages, risks and results of hybrid management of acute trauma patients, and to summarize the cumulative experience from this concept through a review of the currently available English medical literature.

\section{MATERIALS AND METHODS}

A systematic review of the English medical literature was conducted using the Pubmed service of the National Library of Medicine/National Institutes of Health, Cochrane, Embase, and OVID Medline databases to identify all clinical studies regarding hybrid trauma management reported during 2000-2020. Separate search queries were performed using the following terms: "trauma" AND "hybrid" AND "endovascular"; "hybrid emergency room"; "endovascular and hybrid trauma management"; "hybrid operating environment" AND "trauma"; "hybrid operating suite" AND "trauma”; "EVTM"; "RAPTOR” (Resuscitation with Angiography, Percutaneous Techniques and Operative Repair).

Only publications regarding acute trauma patients were included. The following criteria were used to select studies to be included in the analysis: adequate information regarding the mechanism, location, and type of the injury; location and type of hybrid management; surgical intervention; and follow up. Case-reports were excluded from this study as well as clinical studies describing iatrogenic or delayed traumatic event complications. 
Methods of descriptive statistics were used to analyze the investigated studies regarding patients' characteristics; mechanism, location, and type of injury; endovascular and surgical techniques utilized; and outcomes. All continuous variables are presented as means + standard deviation or median with interquartile range. All categorical variables are expressed as percentages.

\section{RESULTS}

The use of hybrid management in acute trauma patients was described in 32 articles, published during 20002020. After exclusion of all case-report descriptive studies (18 studies), 14 studies were included in this review. There were seven case-control studies defined as level III studies and seven case-series defined as level IV studies (Figure 1).

The total number of patients treated in a hybrid emergency room or operating room was 1049, aged 11-79, with an injury severity score ranging between 16 and 75 . Blunt trauma was the leading indication for hybrid trauma management comprising 87.1\% (914/1049) of cases. Penetrating trauma comprised $9.4 \%$ (103/1049) of cases. For 32 cases, the mechanism of trauma was not reported.

Trauma patients were managed in different types of hybrid rooms: $76.6 \%(804)$ of the patients were treated in a hybrid emergency room, $6.2 \%(65)$ of the patients were treated in a hybrid operating theater, $16.1 \%$ (169) of the patients were treated in a RAPTOR suite, and $1.1 \%$ (11) of the patients were treated in a combined computed tomography (CT) and angiography suite with a single pivoting table. Results for case-series studies are presented in Table 1. The hybrid techniques utilized in the reviewed studies included different combinations of laparotomy $-19.3 \%$ (202 patients), thoracotomy $-3.8 \%$ (40 patients), craniotomy $-17.9 \%$ (188 patients), preperitoneal pelvic packing $-1.6 \%$ (17 patients), neck exploration $-1.4 \%$ (15 patients), extremity vascular procedure $-2.6 \%$ (27 patients), transcatheter arterial embolization - 29.7\% (312 patients), resuscitative endovascular balloon occlusion of the aorta $-0.9 \%$ (9 patients), temporary balloon occlusion $-1.1 \%$ (11 patients), and stenting $-5.3 \%$ (56 patients). The preferred site for endovascular procedure was the femoral artery, although this information was not detailed in many of the studies included in this review.

For trauma patients treated in a hybrid room, an overall mortality of $15.2 \%$ (159 patients) was found, ranging between $0 \%$ and $60 \%$ in different studies. For patients treated conventionally, an overall mortality of $27.8 \%$ (239 patients) was found, ranging between $15 \%$ and $47 \%$ in different studies. The reported endovascular procedure-related complications included a retroperitoneal hematoma in one patient and recurrent bleeding in one patient.

Results for case-control studies are presented in Table 2. Hybrid trauma management was associated with a significantly shorter time from patient arrival to intervention in $85.7 \%$ of case-control studies. The mean time to intervention in patients treated in a hybrid room ranged between 45 and $63 \mathrm{~min}$, whereas the mean time to intervention in patients treated conventionally was 64-148 min.

In addition, $42.9 \%$ of the case-control studies demonstrated significantly lower rates of unfavorable outcome for patients treated in a hybrid room as compared with controls. However, the remaining $57.1 \%$ of case-control studies did not report a significant difference in rates of unfavorable outcome. Rates of unfavorable outcome in hybrid trauma management ranged between $15 \%$ and $41 \%$ compared with $22-47 \%$ for patients treated in a conventional room. Unfavorable outcome was defined as in-hospital mortality or 28-day mortality in four and two studies, respectively. One study, reporting results for patients with traumatic brain injury, defined unfavorable outcome as unfavorable functional outcomes at 6 months after injury, as assessed by the Glasgow Outcome Scale-Extended.

Moreover, two studies, which constitute $28.6 \%$ of case-control studies, showed hybrid trauma management was associated with a reduced red blood cell (RBC) transfusion requirement as compared with conventional trauma management. One of these studies [22] reported a mean RBC transfusion volume of 2 units of packed cells for patients treated in a hybrid room, compared with 4 units for patients treated conventionally $(p=0.011)$. The other study [23] reported that the rate of $\mathrm{RBC}$ transfusion requirement for patients treated in a hybrid room and patients treated conventionally was $16 \%$ and $25 \%$ respectively ( $p=0.04$ ).

\section{DISCUSSION}

Following review of the current literature, results suggest possible significant advantages of hybrid trauma management. The survival rate for trauma patients treated in a hybrid room ranged between $40 \%$ and $100 \%$. In addition, the endovascular procedure-related complication rate found in this review, including one patient with a retroperitoneal hematoma and one patient who suffered from recurrent bleeding [24,25], is significantly lower than the complication rate reported in current endovascular literature. For example, Desai et al. reported an overall complication rate of $21 \%$ for patients undergoing endovascular repair of arterial trauma [26]. Similarly, Asaid et al. reported that endovascular repair of traumatic aortic injury was associated with a $20 \%$ complication rate, including common femoral artery thrombosis, access-related vessel thrombosis, and endoleaks [27].

Another promising aspect of hybrid trauma management, found in this review, is a decreased rate of unfavorable outcome, found by $42.9 \%$ of the analyzed case-control studies $[22,28,29]$. However, when looking 


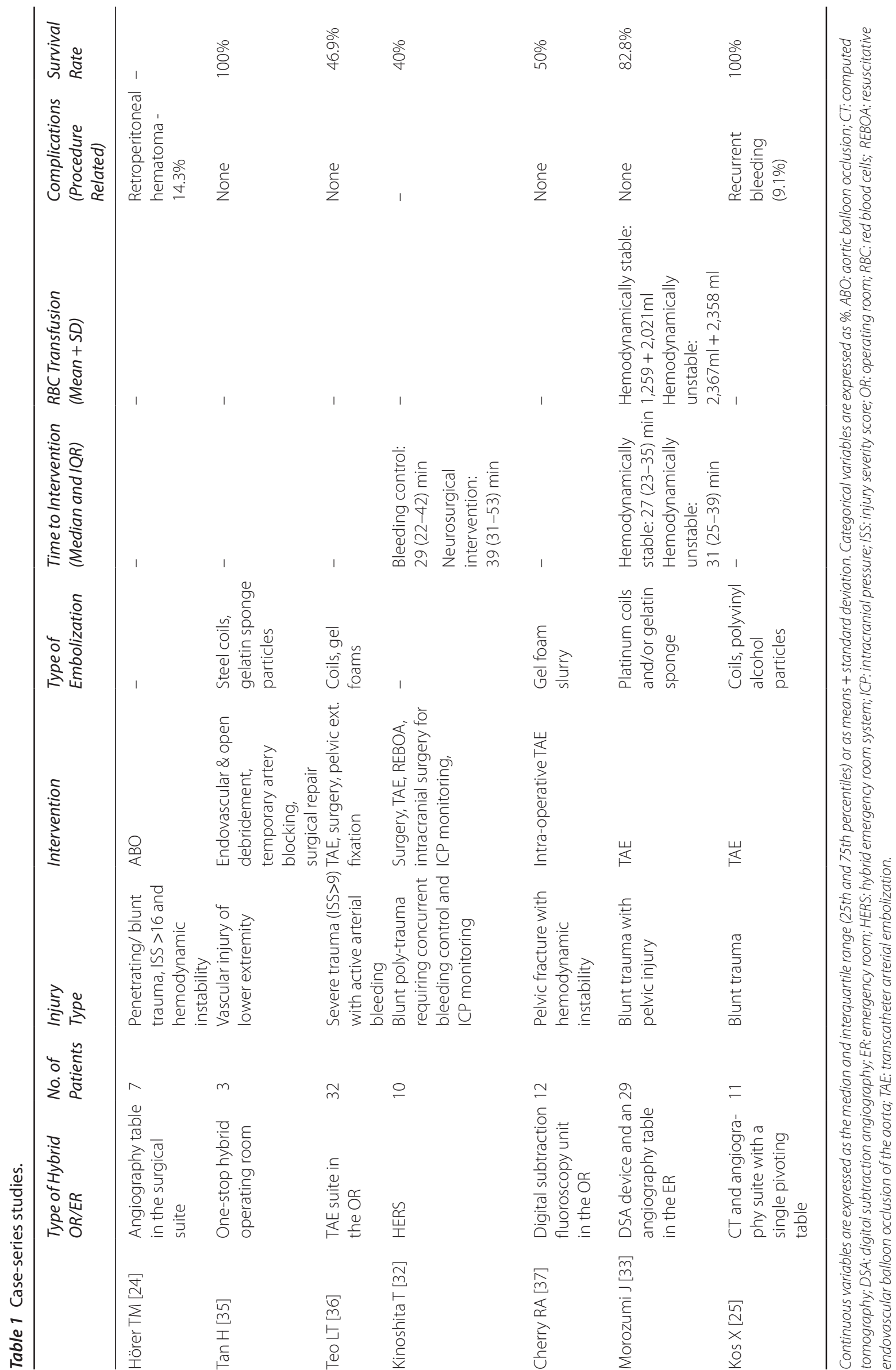




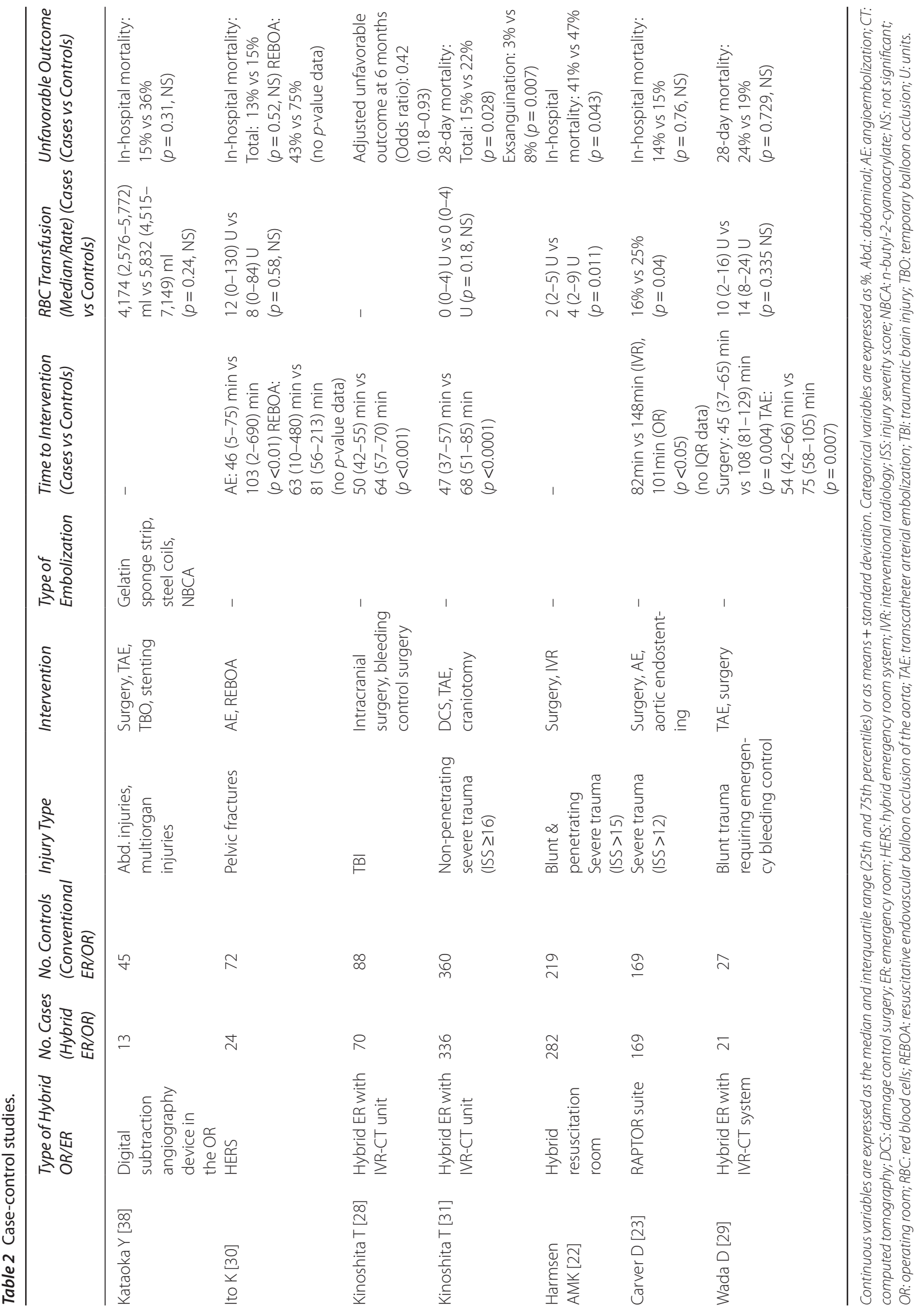


at the ranges of rates of unfavorable outcome in hybrid vs. conventional management $(15-41 \%$ and $22-47 \%$, respectively), the ranges do not appear to be very different. A possible explanation for this finding is that this review includes studies with a wide range of injury type and severity level. Thus, rates of unfavorable outcome are highly variable across different studies included in this review, making comparison of the studies very limited. In addition, this review has found a reduced RBC transfusion volume requirement in patients treated in a hybrid room, reported in two of the studies analyzed $[22,23]$. These findings may be partially attributed to the significantly shorter time from patient arrival to intervention for patients treated in a hybrid room as compared with conventional management, as reported in some of the studies reviewed [23,28-31].

An interesting aspect of our review is that it revealed a wide range of different types of hybrid rooms used for trauma management. In several studies, the type of hybrid emergency room used for trauma management was a hybrid emergency room system (HERS) which consists of a trauma resuscitation room equipped with a CT scanner, fluoroscopy equipment, and an operating room setup [28,30-32]. The HERS was installed in Osaka, Japan in 2011 and was first reported by the founding members of the Japanese Association for Hybrid Emergency Room System [17]. The HERS is equipped with a sliding CT scanner system with interventional radiology features and was created in order to facilitate both diagnostic and therapeutic interventions in a single room [17]. One study included in this review reported the use of an intensive care unit designed for surgical intervention and equipped with a mobile digital subtraction angiography device [33]. In two other studies, the hybrid emergency room used for trauma management was a RAPTOR suite $[22,23]$. The new concept and establishment of a RAPTOR suite was first described by Kirkpatrick et al. [34]. This suite, which entered clinical service in March 2013, was designed to provide the ultimate setting to prevent exsanguination and eliminate delays in hemorrhage control in trauma patients. This pioneer suite was equipped with a ceiling mounted single-planar angiography, coupled with a hybrid surgical operating table, and integrated with an operating room integration system [34]. Other hybrid operating rooms used for trauma management were operating rooms equipped with a digital subtraction angiography device [24,35-38]. One study included in this review, reported results of trauma patients' management in an angiography suite equipped with a spiral CT [25].

These new hybrid rooms present exciting new possibilities for the management of trauma patients. On the other hand, the benefits of hybrid trauma management must be weighed against the high cost and human resource demand of these rooms and workflows [20]. Furthermore, the limited availability of hybrid rooms should be taken into consideration since hybrid rooms are used not only for trauma management, but for a wide range of procedures as well, such as treatment of neurovascular disease [8], which is mainly performed in elective surgery settings. Similarly, new approaches for aortic valve replacement utilize hybrid operating rooms in some medical centers and are also performed electively [10]. Therefore, these hybrid rooms and the operating team needed to operate them may not be available for trauma patients on a $24 \mathrm{~h}$ basis.

While the results of the reviewed studies are promising, a number of limitations of this review must be highlighted. One important limitation is the fact that all case-control studies have compared management of trauma patients in a single center before and after installation of a hybrid room. It is important to recognize that throughout the years, other than the installation of a hybrid room, many other changes must have been implemented in these medical centers alongside worldwide advancements in trauma care. Therefore, the promising results of these studies may reflect not only the advantages of installation and utilization of hybrid rooms, but also the different improvements which have evolved in the care of trauma patients.

Another limitation is the retrospective nature of the reviewed studies, which are therefore subject to selection bias and information bias. When discussing our results, it is important to note that no randomized controlled or prospective studies were found. Thus, this review included studies with a level of evidence of III and IV, and no level I or II studies were analyzed.

Lastly, limited comparability of the analyzed studies must be taken into account. The reviewed studies differ greatly with regard to patients' injury type and severity. Furthermore, this review included studies performed in different centers worldwide. Hence, the differences in therapeutic approach, capabilities, and level of expertise between different trauma centers must be taken into consideration.

\section{CONCLUSIONS}

The existing studies show promising results regarding the outcomes of hybrid trauma management, including decreased rates of unfavorable outcome, shorter time from arrival to treatment, and reduced requirement for blood transfusion in different studies. However, the published studies to date are observational and retrospective studies with a low level of evidence. Interestingly, some centers have already adopted hybrid trauma management as a standard of care. Future data collected from these centers may further support the use of this approach in the future as a standard of care worldwide.

We believe findings of this review justify the urgent need for further prospective studies to better understand the possible advantages of and indications for hybrid trauma management. These future studies should also 
address the high cost and resource demand of this promising new approach.

\section{Ethics Statement}

(1) All the authors mentioned in the manuscript have agreed to authorship, read and approved the manuscript, and given consent for submission and subsequent publication of the manuscript.

(2) The authors declare that they have read and abided by the JEVTM statement of ethical standards including rules of informed consent and ethical committee approval as stated in the article.

\section{Conflicts of Interest}

We wish to confirm that there are no known conflicts of interest associated with this publication and there has been no financial support for this work.

\section{Funding}

This research did not receive any grant or funding.

\section{Author Contributions}

MP was responsible for the drafting of the manuscript, data acquisition, data analysis and interpretation. BK was responsible for study conception and design, data analysis and interpretation, critical revision.

\section{REFERENCES}

[1] Pryor JP, Braslow B, Reilly PM, Gullamondegi O, Hendrick JH, Schwab CW. The evolving role of interventional radiology in trauma care. J Trauma. 2005;59(1): 102-4.

[2] Zealley IA, Chakraverty S. The role of interventional radiology in trauma. BMJ. 2010;340:c497.

[3] Ball CG, Kirkpatrick AW, D'Amours SK. The RAPTOR: Resuscitation with angiography, percutaneous techniques and operative repair. Transforming the discipline of trauma surgery. Can J Surg. 2011;54(5):E3-4.

[4] Branco BC, DuBose JJ, Zhan LX, et al. Trends and outcomes of endovascular therapy in the management of civilian vascular injuries. J Vasc Surg. 2014;60(5):1297307.e1.

[5] Branco BC, Boutrous ML, DuBose JJ, et al. Outcome comparison between open and endovascular management of axillosubclavian arterial injuries. J Vasc Surg. 2016;63(3):702-9.

[6] Romagnoli AN, Zeeshan M, Joseph B, Brenner ML. Utilization of endovascular and open surgical repair in the United States: A 10-year analysis of the National Trauma Databank (NTDB). Am J Surg. 2019;218(6): 1128-33.

[7] Jeon HJ, Lee JY, Cho BM, Yoon DY, Oh S-M. Four-year experience using an advanced interdisciplinary hybrid operating room: Potentials in treatment of cerebrovascular disease. J Korean Neurosurg Soc. 2019;62(1): 35-45.

[8] Choi E, Lee JY, Jeon HJ, Cho B-M, Yoon DY. A hybrid operating room for combined surgical and endovascular procedures for cerebrovascular diseases: A clinical experience at a single centre. Br J Neurosurg. 2019;33(5):490-4.

[9] Singh A, Mehta Y, Parakh R, Kohli V, Trehan N. Role of hybrid operating room in surgery for the right atrial thrombus, pulmonary thrombi, and ventricular septal rupture after myocardial infarction. Ann Card Anaesth. 2016;19(4):717-21.

[10] Spaziano M, Lefèvre T, Romano M, et al. Transcatheter aortic valve replacement in the catheterization laboratory versus hybrid operating room: Insights from the FRANCE TAVI registry. JACC Cardiovasc Interv. 2018; 11(21):2195-203.

[11] Richter PH, Yarboro S, Kraus M, Gebhard F. One year orthopaedic trauma experience using an advanced interdisciplinary hybrid operating room. Injury. 2015;46 Suppl 4:S129-34.

[12] Hörer TM, Pirouzram A, Khan M, et al. Endovascular Resuscitation and Trauma Management (EVTM) - practical aspects and implementation. Shock. 2020; In Press. doi: 10.1097/SHK.0000000000001529

[13] Karkos CD, Mair R, Markose G, Fishwick G, London NJM, Naylor, AR. Hybrid procedures combining open and endovascular surgical techniques for the management of subclavian artery injuries. J Trauma. 2007;63(5): E107-10.

[14] Bilos L, Pirouzram A, Toivola A, Vidlund M, Cha SO, Hörer T. EndoVascular and Hybrid Trauma Management (EVTM) for blunt innominate artery injury with ongoing extravasation. Cardiovasc Intervent Radiol. 2017;40(1):130-4.

[15] Schmid FX, Philipp A, Link J, Zimmerman M, Birnbaum DE. Hybrid management of aortic rupture and lung failure: Pumpless extracorporeal lung assist and endovascular stent-graft. Ann Thorac Surg. 2002;73(5):1618-20.

[16] Wada D, Hayakawa K, Maruyama S, et al. A paediatric case of severe tracheobronchial injury successfully treated surgically after early CT diagnosis and ECMO safely performed in the hybrid emergency room. Scand J Trauma Resusc Emerg Med. 2019;27(1):49.

[17] Founding members of the Japanese Association for Hybrid Emergency Room System (JA-HERS). The hybrid emergency room system: A novel trauma evaluation and care system created in Japan. Acute Med Surg. 2019;6(3):247-51.

[18] Watanabe H, Shimojo Y, Hira E, et al. First establishment of a new table-rotated-type hybrid emergency room system. Scand J Trauma Resusc Emerg Med 2018;26(1):80.

[19] Wada D, Nakamori Y, Kanayama S, et al. First installation of a dual-room IVR-CT system in the emergency room. Scand J Trauma Resusc Emerg Med. 2018;26:17.

[20] Fehr A, Beveridge J, D’Amours SD, Kirkpatrick AW, Ball CG. The potential benefit of a hybrid operating environment among severely injured patients with persistent hemorrhage: How often could we get it right? J Trauma Acute Care Surg. 2016;80(3):457-60.

[21] Tatum D, Duchesne J, Pereira B, et al. Time to hemorrhage control in a hybrid ER system: Is it time to 
change? Shock. 2020; In Press. doi: 10.1097/ SHK.0000000000001539.

[22] Harmsen AMK, Giannakopoulos GF, Terra M, de Lange de Klerk ESM, Bloemers FW. Ten year maturation period in a level-I trauma center, a cohort comparison study. Eur J Trauma Emerg Surg. 2017;43(5): 685-90.

[23] Carver D, Kirkpatrick AW, D’Amours S, Hameed SM, Beveridge J, Ball CG. A prospective evaluation of the utility of a hybrid operating suite for severely injured patients: Overstated or underutilized? Ann Surg. 2020; 271(5):958-61.

[24] Hörer TM, Skoog P, Pirouzram A, Nilsson KF, Larzon T. A small case series of aortic balloon occlusion in trauma: Lessons learned from its use in ruptured abdominal aortic aneurysms and a brief review. Eur J Trauma Emerg Surg. 2016;42(5):585-92.

[25] Kos X, Fanchamps JM, Trotteur G, Dondelinger RF. Radiologic damage control: Evaluation of a combined CT and angiography suite with a pivoting table. Cardiovasc Intervent Radiol. 1999;22(2):124-9.

[26] Desai SS, DuBose JJ, Parham CS, et al. Outcomes after endovascular repair of arterial trauma. J Vasc Surg. 2014;60(5):1309-14.

[27] Asaid R, Boyce G, Atkinson N. Endovascular repair of acute traumatic aortic injury: experience of a level-1 trauma center. Ann Vasc Surg. 2014;28(6):1391-5.

[28] Kinoshita T, Hayashi M, Yamakawa K, et al. Effect of the hybrid emergency room system on functional outcome in patients with severe traumatic brain injury. World Neurosurg. 2018;118:e792-9.

[29] Wada D, Nakamori Y, Yamakawa K, Fujimi S. First clinical experience with IVR-CT system in the emergency room: positive impact on trauma workflow. Scand J Trauma Resusc Emerg Med. 2012;20:52.

[30] Ito K, Nagao T, Tsunoyama T, et al. Hybrid emergency room system improves timeliness of angioembolization for pelvic fracture. J Trauma Acute Care Surg. 2020; 88(2):314-9.

[31] Kinoshita T, Yamakawa K, Matsuda H, et al. The survival benefit of a novel trauma workflow that includes immediate whole-body computed tomography, surgery, and interventional radiology, all in one trauma resuscitation room: A retrospective historical control study. Ann Surg. 2019;269(2):370-6.

[32] Kinoshita T, Yamakawa K, Yoshimura J, et al. First clinical experiences of concurrent bleeding control and intracranial pressure monitoring using a hybrid emergency room system in patients with multiple injuries. World J Emerg Surg. 2018;13:56.

[33] Morozumi J, Homma H, Ohta S, et al. Impact of mobile angiography in the emergency department for controlling pelvic fracture hemorrhage with hemodynamic instability. J Trauma. 2010;68(1):90-5.

[34] Kirkpatrick AW, Vis C, Dubé M, et al. The evolution of a purpose designed hybrid trauma operating room from the trauma service perspective: the RAPTOR (Resuscitation with Angiography Percutaneous Treatments and Operative Resuscitations). Injury. 2014;45(9):1413-21.

[35] Tan H, Zhang LY, Guo QS, et al. "One-stop hybrid procedure" in the treatment of vascular injury of lower extremity. Indian J Surg. 2015;77(1):75-8.

[36] Teo LT, Punamiya S, Chai CY, et al. Emergency angio-embolisation in the operating theatre for trauma patients using the C-Arm digital subtraction angiography. Injury. 2012;43(9):1492-6.

[37] Cherry RA, Goodspeed DC, Lynch FC, Delgado J, Reid SJ. Intraoperative angioembolization in the management of pelvic-fracture related hemodynamic instability. J Trauma Manag Outcomes. 2011;5:6.

[38] Kataoka Y, Minehara H, Kashimi F, et al. Hybrid treatment combining emergency surgery and intraoperative interventional radiology for severe trauma. Injury. 2016;47(1):59-63. 